Published in final edited form as:

J Urol. 2016 September ; 196(3): 703-708. doi:10.1016/j.juro.2016.03.152.

\title{
Clinical Findings and Treatment Outcomes in Patients with Extraprostatic Extension Identified on Prostate Biopsy
}

\author{
Katherine Fleshner ${ }^{1}$, Melissa Assel ${ }^{2}$, Nicole Benfante ${ }^{3}$, Justin Lee ${ }^{3}$, Andrew Vickers $^{2}$, \\ Samson Fine ${ }^{4}$, Sigrid Carlsson ${ }^{5,6}$, and James Eastham ${ }^{3}$ \\ ${ }^{1}$ McGill University, Montreal, Canada \\ ${ }^{2}$ Department of Epidemiology \& Biostatistics, Memorial Sloan Kettering Cancer Center, New York, \\ USA
}

${ }^{3}$ Urology Service at the Department of Surgery, Memorial Sloan Kettering Cancer Center, New York, USA

${ }^{4}$ Department of Pathology, Memorial Sloan Kettering Cancer Center, New York, USA

${ }^{5}$ Department of Surgery, Memorial Sloan Kettering Cancer Center, New York, USA

${ }^{6}$ Department of Urology, Institute of Clinical Sciences, Sahlgrenska Academy at University of Göteborg, Sweden

\section{Abstract}

Purpose-To describe the histopathological, clinical and imaging findings among men with extraprostatic extension (EPE) on prostate biopsy.

\begin{abstract}
Materials and methods-We searched our Institutional Pathology Database, between the years 2004-2015, for pathology reports detailing EPE on prostate biopsy in untreated patients. Patient characteristics, biopsy features, imaging interpretations and outcomes were examined.
\end{abstract}

Results-Of 19,950 patients with prostate cancer on biopsy, 112 had EPE, a prevalence of $0.6 \%$ (95\% CI 0.5\%-0.7\%). Most patients had palpable, high-grade (Gleason score 9), high-volume, disease classified as high-risk $(30 \% ; 34 / 112)$, locally advanced $(15 \% ; 17 / 112)$, or metastatic $(35 \%$;

Corresponding author: Dr. James Eastham, Urology Service at the Department of Surgery, Memorial Sloan Kettering Cancer Center, 353 E68th St, New York, NY, USA, Phone: 646-422-4322, easthamj@ mskcc.org.

Conflict of interest: Andrew Vickers is named on a patent application for a statistical method to detect prostate cancer. This has been commercialized by Opko Health and Andrew Vickers receives royalties from any sales of the test. Andrew Vickers is on the advisory board of Opko Health and has Opko stock options. Andrew Vickers has a consulting or advisory role in Genomic DX and Genomic Health. No other author has any conflict of interest to declare.

Abstract submission: Parts of this manuscript has been submitted as an abstract to the upcoming American Urological Association (AUA) annual meeting in San Diego in May, 2016 and selected to be presented as a moderated poster.

Author contributions:

Conception and design: JE, AV

Data acquisition: KF, JE, NB, SC

Data analysis and interpretation: KF, SC, AV, JE, SF, MA

Drafting the manuscript: KF, SC

Critical revision of the manuscript for scientific and factual content: All authors.

Statistical analysis: KF, SC, MA

Supervision: JE, SC, AV

Other: SF participated in the pathology review of the prostate biopsies 
39/112). Most had one or two cores with EPE, typically at the base and with concomitant perineural invasion. EPE was identified by MRI in $80 \%$ (32/40). The median follow-up for those who did not die was 1.3 years (IQR 0.3, 4.2). Outcomes for the subgroup of 24 men who had an RP were consistent with high risk disease: positive margins (58\%; 14/24), SVI (42\%; 10/24), and LNI $(46 \% ; 11 / 24)$. For the entire cohort, the 3-year risks of metastasis and overall mortality were $32 \%$ (95\% CI 22\%, 44\%) and 37\% (95\% CI 27\%, 50\%), respectively. We did not find evidence to suggest that the proportion of cores with cancer that also had EPE was associated with overall mortality $(\mathrm{p}=0.09)$.

Conclusions-EPE is a rare finding on prostate biopsy. It is strongly associated with other features of aggressive prostate cancer.

\section{Keywords}

extraprostatic extension; prostate cancer; prostate biopsy; radical prostatectomy

\section{Introduction}

Extraprostatic extension (EPE) on histopathological examination refers to the identification of tumor cells beyond the borders of the prostate, most often recognized as tumor intermingling with periprostatic adipose tissue. EPE can manifest focally throughout the tissue, that is, only a few neoplastic glands are seen outside the prostate, or become nonfocal with more glands ${ }^{1}$. Although most commonly detected in the radical prostatectomy (RP) specimen, EPE can also be found on preoperative Magnetic Resonance Imaging (MRI) and on prostate biopsy ${ }^{2}$.

EPE in the RP specimen is well-studied and is a critical part of the pathological tumor staging process, as all RP specimens containing EPE are classified as pathological stage $\mathrm{pT}^{3}$. EPE found at RP is a risk factor for poor prognosis and is therefore often included in postoperative nomograms predicting outcomes such as biochemical recurrence (BCR) after $\mathrm{RP}^{1,4}$. Sometimes adjuvant radiation therapy (RT) is recommended upon detection ${ }^{5}$.

EPE can also sometimes be captured on MRI, used for staging purposes and treatment planning. MRI for detecting EPE at surgical pathology has a moderate sensitivity and specificity, $72 \%$ and $65 \%$, respectively 6 .

Occasionally, EPE is also noted on the pathology report from the prostate biopsy. However, its detection on the prostate biopsy is fairly uncommon. To the best of our knowledge, there is only one publication in the literature reporting outcomes of patients with EPE on prostate biopsy. In that study, conducted at Johns Hopkins University (JHU), Miller et al examined 51,891 biopsies performed between 1997 and 2009 and reported a prevalence of EPE of only $0.19 \%{ }^{2}$. Because of its rare occurrence, the clinical significance of this finding is not well understood. Little is known whether biopsy-detected EPE is an independent predictor of aggressive prostate cancer.

We therefore undertook the present study to first, estimate the frequency of this occurrence among men who had a positive prostate biopsy at our institution, and second, describe the 
histopathological and clinical characteristics, as well as outcomes. Third, we sought to compare the congruity of EPE detection on biopsy, MRI and RP specimens. We hypothesized that with the current transrectal ultrasound (TRUS)-guided biopsy technique, EPE would mainly be found at the base of the prostate.

\section{Materials and Methods}

\section{Selection Criteria}

The present study was undertaken following Institutional Review Board approval. Figure 1 depicts the flowchart for the studied cohort. Between inception of the MSKCC institutional electronic pathology database in 2004 and last follow-up July 31 2015, a total of 19,950 men with prostate biopsies positive for prostate cancer were recorded. Patients were seen either in the urology clinic or by medical oncology or radiation oncology at our institution.

A free-text search for the words ( ( extracapsular OR extraprostatic OR adipose ) AND ( NOT no extracapsular OR NOT no extraprostatic) ) within prostate biopsy pathology reports performed during this time period retrieved 206 hits. Because tumor in the adipose tissue is per definition EPE, and intraprostatic adipose tissue is extremely rare, we also included the word "adipose". Pathology reports with "benign adipose tissue" and ineligible pathology reports caught by the search were excluded.(Figure 1)

Since the irradiated or treated prostate is difficult to examine under the microscope, and because our study questions relate to men without prior treatment, we excluded men who had a date of therapy preceding the date of biopsy (androgen deprivation therapy (ADT), radiotherapy, brachytherapy, high intensity focused ultrasound). We also excluded men who had a date of RP preceding the date of biopsy, who had a biopsy of the prostate bed upon recurrence. This left a final count of 112 men for analysis (Figure 1) and 183 biopsy areas for patients with multiple biopsies with EPE.

Since our institution is a referral center, the pathology report search identified several types of biopsies: those representing new biopsies performed at our institution, repeat biopsies seeking to corroborate the prostate cancer diagnosis from an outside institution, and rereviewed reports by our pathologists of diagnostic biopsies performed at outside institutions. Patients presenting with distant metastasis that had confirmatory biopsies could thus be included in the search.

\section{Statistical Analysis}

The frequency of EPE on prostate biopsy was calculated as the prevalence of EPE from the pathology report search divided by the total number of men with biopsies positive for prostate cancer during the study period, excluding patients with prior treatment and those with ineligible reports. A $95 \%$ confidence interval around this proportion was calculated using the binomial distribution.

For our aim to describe the histopathological and clinical characteristics, we applied descriptive statistics; frequencies and proportions for categorical variables, and medians and interquartile ranges (IQR) for continuous variables. For tumor risk grouping, we used the 
National Comprehensive Cancer Network classification: Low risk: T0, T1c, T2a and GS $\leq 6$ and PSA < 10; Intermediate risk: T2b, T2c or GS 7 or PSA 10-20; High risk: T3a or GS 8-10 or PSA 220 ; Locally advanced: T3b, T3c, T4; Metastatic: N1 or M1 ${ }^{7}$. Because there can be a delay in imaging or evaluation for metastatic disease, we also classified men who were diagnosed with confirmed distant metastasis within 3 months from biopsy as M1.

Initial treatments were defined as the primary treatment administered within one year following diagnosis, provided that the patient was followed for at least one year. Patients receiving neoadjuvant ADT, were classified according to their active treatment; e.g. ADT plus RT was categorized as RT.

The following pathologic outcomes were reported for a subgroup of 24 men who underwent RP: EPE at RP, positive surgical margins, seminal vesicle invasion (SVI) and lymph node invasion (LNI). Upgrading at the time of RP, or risk of biochemical recurrence, could not be examined due to small sample/event size.

To describe the clinical course for all patients, we calculated the cumulative risk of metastasis and death using the Kaplan-Meier method. Since certain prostate cancer treatments may in theory increase risk of cardiovascular side-effects and competing mortality, we report overall mortality as the outcome, as opposed to prostate cancer mortality, not to risk underreporting mortality. Last, we performed a Cox regression analysis to study the association between the log-transformed proportion of cores with cancer that also had EPE and overall mortality

For our third aim to compare the congruity of EPE detection on biopsy and MRI, we calculated the proportion of men with positive MRIs whose impression fields mentioned EPE, when EPE was present on prostate biopsy. We selected MRI imaging (16 MRI, 21 endorectal MRI, 2 MR-spectroscopy imaging/MRSI, and 1 MRI-TRUS-fusion) performed within a six month window around the time of biopsy that found EPE and excluded 3 men who had MRI post-RP. Last, we noted the location of EPE on biopsies and compared it to that reported on the MRI (right or left side).

\section{Results}

Of the 19,950 patients with a positive biopsy, 112 reported EPE, a prevalence of $0.6 \%$ (95\% CI $0.5 \%-0.7 \%$ ). Patient characteristics for the final cohort of 112 patients are described in Table 1. The median age was 68 years and the median PSA level prior to biopsy was 15.9 $\mathrm{ng} / \mathrm{mL}$ (IQR; 8.1, 62.3). The patients in the cohort had largely high grade disease (Gleason score 9) and most were classified as high risk or had locally advanced or metastatic disease. Patients received a variety of treatment combinations, with the first treatment within the first year distributed as follows: 42/112 (38\%) ADT, 23/112 (21\%) RP and 23/112 (21\%) RT; the remaining were not followed for a year or received no treatment within the first year (of which one man had a salvage RP). Treatments administered appeared risk group appropriate, but heterogeneously distributed (Figure 2).

The median follow-up for patients who did not develop mets was 1.3 years (IQR 0.3, 4.2) and similar for those who did not die; 1.3 years. For the entire cohort of men with EPE, the 
3-year risks of metastasis and overall mortality were $32 \%$ (95\% CI 22\%, 44\%) and 37\% (95\% CI 27\%, 50\%), respectively. (Figure 3)

A total of 24 patients in the cohort underwent RP. Of these, 10/24 (42\%) were preoperatively classified as intermediate risk, $12 / 24(50 \%)$ as high risk and 2/24 (8\%) as metastatic. At the time of RP 21/24 (88\%) patients had non-focal EPE, 2/24 (8\%) had focal EPE and 1/24 (4\%) patient had no EPE. Patients had outcomes after RP consistent with high risk, such as positive margins 14/24 (58\%), SVI 10/24 (42\%) and LNI 11/24 (46\%).

With regards to biopsy (Table 3), most patients underwent a 14 core biopsy with a median number of cores with cancer of 10 (IQR; 7, 13), i.e. a median of $86 \%$ cores cancer (IQR $57 \%, 100 \%)$. Most patients had only one or two cores with EPE and the median length of cancer per core with EPE was 12 millimeters (IQR 8, 15). In a per core analysis, simultaneous perineural invasion was detected in 62\% (113/183) of cores with EPE. The majority of cores with EPE were graded as Gleason score 8-10. Most of the EPE was found at the base of the prostate $(37 \% ; 67 / 183)$.

The proportion of patients with MRI positive for EPE when EPE was present on biopsy was $80 \%$ (32/40) (95\% CI 67\%-93\%). In 58\% (23/40) (95\% CI 41-74\%) of men with MRI, the MRI was read as EPE on the same side/-s as the biopsy/ies with EPE. In 10\% (4/40) did the MRI identify EPE on one side but not the other.

On univariate Cox regression analysis, we did not find sufficient evidence to suggest that the proportion of cores with cancer that also had EPE was associated with overall mortality $(\mathrm{p}=0.09)$.

\section{Discussion}

We sought to describe a cohort of men at our institution who had EPE on the prostate biopsy in an attempt to elucidate the clinical significance of this finding. We found that many of the men presented with other features of adverse disease such as high PSA levels and high Gleason score. Most were classified as high risk, and patients who exhibited features of intermediate and high risk disease preoperatively had outcomes after RP consistent with high risk, such as EPE, SVI and positive lymph nodes. This suggests that EPE on the biopsy likely accompanies other adverse features that collectively constitute aggressive or advanced prostate cancer.

The sparse data in the literature on the prevalence and understanding of EPE on biopsy has seemingly rendered it devoid of influence in patient care. Current preoperative nomograms do not incorporate EPE into their prediction models because its presence is rarely confirmed; biopsy capture is infrequent and MRI is not sufficiently sensitive. We did not find sufficient evidence to suggest that the frequency of EPE was independently associated with overall mortality.

The present study adds to the literature, and our data corroborate the prior study from JHU in which EPE on biopsy was similarly strongly associated with extensive, high-grade disease $^{2}$. Results were concordant: most common Gleason score in cores with EPE 9 
(MSKCC) vs. 8 (JHU); \% cancer in biopsy cores $86 \%$ (median) vs. $70 \%$ (mean); concomitant perineural invasion $62 \%$ vs. $75 \%$; 3 -year risk of metastasis $32 \%$ vs. $40 \%$; and outcomes after RP were similar: EPE ( $96 \%$ vs. $91 \%$ ); positive margins ( $58 \%$ vs. $55 \%$ ); SVI (52\% vs. $45 \%)$ and LNI $(46 \% \text { vs. } 10 \%)^{2}$

In the present study, we also sought to investigate how EPE detection on biopsy affected RP outcomes. Despite the pathology reports documenting EPE prior to RP, most patients had positive margins (58\%), SVI (42\%) and LNI (46\%). These rates are in line with, or even higher than those previously reported from our institution for patients with preoperative high risk; Imnadze et al reported; positive margins (27\%), SVI (22\%) and LNI $(23 \%)^{8}$. It is probable that the high prevalence of adverse outcomes in our cohort is a consequence of the high risk nature of many of the patients in the cohort; it is likely that a small and focused biopsy needle picking up EPE is catching only a small area of an underlying larger tumor of advanced disease (tip of the iceberg).

Another feature that highlighted the aggressive disease found among our cohort was that $88 \%$ of RP specimens in our cohort contained non-focal $\mathrm{EPE}^{9}$, i.e. EPE was found in more than a few small foci. Non-focal EPE indicates a greater tumor volume beyond the confines of the prostate and it is known to be more aggressive and carry a higher risk of BCR compared to focal EPE ${ }^{4,9-10}$.

Imaging technology such as MRI, which maps EPE in a three-dimensional and directional manner, may continue to be a useful adjunct in preoperative planning alongside the pathology report from the needle biopsy. In $80 \%$ of MRIs, the MRI was read as having EPE, which is in line with a prior study by Rud et al. which reported a sensitivity of $72 \%{ }^{6}$. In comparison, $96 \%$ of RP specimens also contained EPE, which corroborates a study by Ravery et al. that reported a positive predictive value of EPE on biopsy of $94 \%{ }^{11}$.

As expected, EPE at biopsy was mainly found in the posterolateral areas of the prostate. Concomitant perineural invasion was detected in $62 \%$ of cores with EPE, which is similarly expected, since perineural growth is a known major route of $\mathrm{EPE}^{1}$.

Moreover, the current method of performing biopsies does not specifically seek out the presence of EPE; rather, it is a serendipitous finding. Similarly, reporting presence or absence of EPE on biopsy may not be routine among pathologists and may be described in different terminology (e.g. extraprostatic extension, extracapsular extension, tumor intermingling with adipose tissue); a study by Bryant et al. exploring interobserver variability of recognizing EPE on the RP specimen reported a lack of consensus among several pathologists in $32 \%$ of cases ${ }^{12}$. Taken together, this may account for the low prevalence.

The present study is not devoid of limitations. Most notably, it is difficult to characterize a poorly understood feature of disease with a small number of patients; yet, to the best of our knowledge, it is the largest series in the scientific literature this far. In comparison to the study by Miller et al, who reported a prevalence of EPE on biopsy of $0.19 \%^{2}$, we found a prevalence of $0.6 \%$. Our finding of a higher prevalence may be explained by the fact that our institution is a referral center, thus reflecting a selection of patients with more aggressive 
disease who may be more likely to harbor EPE compared to the general population.

Considering the variety of terms used to denote EPE, it is also possible that our search within the pathology reports missed some EPE cases. Finally, the MRI analyses of EPE were reliant on an "impressions" field in our database that briefly described what was seen on the films. A more accurate method would be to have radiologists independently re-review the MRIs for presence and location of EPE.

\section{Conclusion}

In conclusion, EPE on prostate biopsy is a rare occurrence. When detected, it is associated with other features of aggressive prostate cancer.

\section{Acknowledgments}

Funding: SC is supported by a grant from AFA Insurance. Other grant support received from David H. Koch provided through the Prostate Cancer Foundation, the Sidney Kimmel Center for Prostate and Urologic Cancers, P50-CA92629 SPORE grant from the National Cancer Institute to Dr. H Scher, and the P30-CA008748 NIH/NCI Cancer Center Support Grant to MSKCC.

\section{References}

1. Fine SW, Amin MB, Berney DM, et al. A contemporary update on pathology reporting for prostate cancer: biopsy and radical prostatectomy specimens. Eur Urol. 2012; 62:20-39. [PubMed: 22421083]

2. Miller JS, Chen Y, Ye H, Robinson BD, Brimo F, Epstein JI. Extraprostatic extension of prostatic adenocarcinoma on needle core biopsy: report of 72 cases with clinical follow-up. BJU Int. 2010; 106:330-3. [PubMed: 20002671]

3. Magi-Galluzzi C, Evans AJ, Delahunt B, et al. International Society of Urological Pathology (ISUP) Consensus Conference on Handling and Staging of Radical Prostatectomy Specimens. Working group 3: extraprostatic extension, lymphovascular invasion and locally advanced disease. Mod Pathol. 2011; 24:26-38. [PubMed: 20802467]

4. Ball MW, Partin AW, Epstein JI. Extent of extraprostatic extension independently influences biochemical recurrence-free survival: evidence for further pT3 subclassification. Urology. 2015; 85:161-4. [PubMed: 25440818]

5. Theiss M, Wirth MP, Manseck A, Frohmuller HG. Prognostic significance of capsular invasion and capsular penetration in patients with clinically localized prostate cancer undergoing radical prostatectomy. Prostate. 1995; 27:13-7. [PubMed: 7603912]

6. Rud E, Klotz D, Rennesund K, Baco E, et al. Preoperative magnetic resonance imaging for detecting uni- and bilateral extraprostatic disease in patients with prostate cancer. World J Urol. 2015; 33:1015-21. [PubMed: 25059766]

7. Mohler JL, Kantoff PW, Armstrong AJ, et al. Prostate cancer, version 2.2014. J Natl Compr Canc Netw. 2014; 12:686-718. [PubMed: 24812137]

8. Imnadze M, Sjoberg DD, Vickers AJ. Adverse Pathologic Features at Radical Prostatectomy: Effect of Preoperative Risk on Oncologic Outcomes. Eur Urol. 2015 In press.

9. Epstein JI, Carmichael MJ, Pizov G, Walsh PC. Influence of capsular penetration on progression following radical prostatectomy: a study of 196 cases with long-term followup. J Urol. 1993; 150:135-41. [PubMed: 7685422]

10. Jeong BC, Chalfin HJ, Lee SB, et al. The relationship between the extent of extraprostatic extension and survival following radical prostatectomy. Eur Urol. 2015; 67:342-6. [PubMed: 24968968]

11. Ravery V, Boccon-Gibod LA, Dauge-Geffroy MC, et al. Systematic biopsies accurately predict extracapsular extension of prostate cancer and persistent/recurrent detectable PSA after radical prostatectomy. Urology. 1994; 44:371-6. [PubMed: 7521093] 
12. Bryant RJ, Schmitt AJ, Roberts IS, et al. Variation between specialist uropathologists in reporting extraprostatic extension after radical prostatectomy. J Clin Pathol. 2015; 68:465-72. [PubMed: 25792751] 


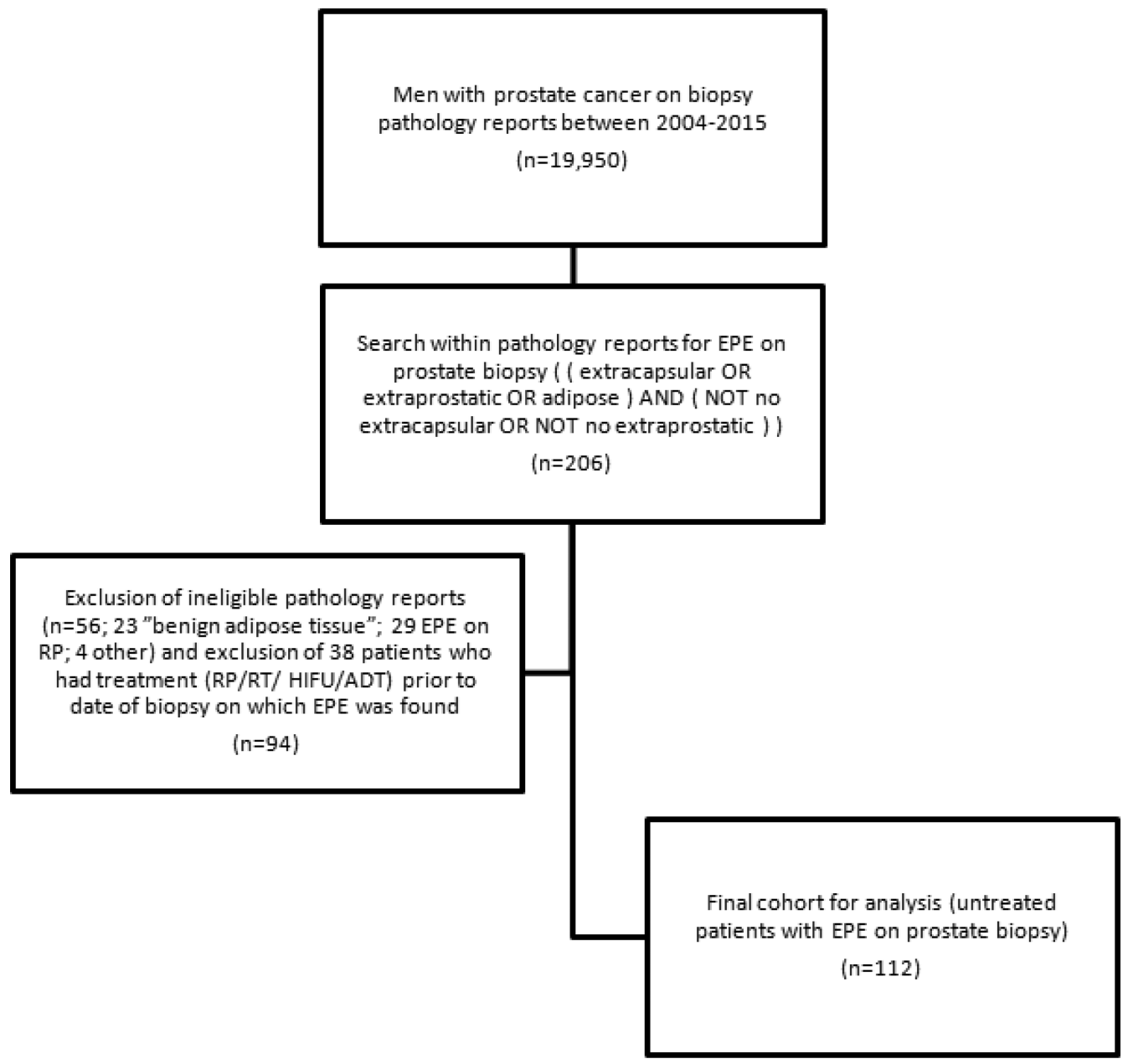

Figure 1.

Flow chart of cohort 


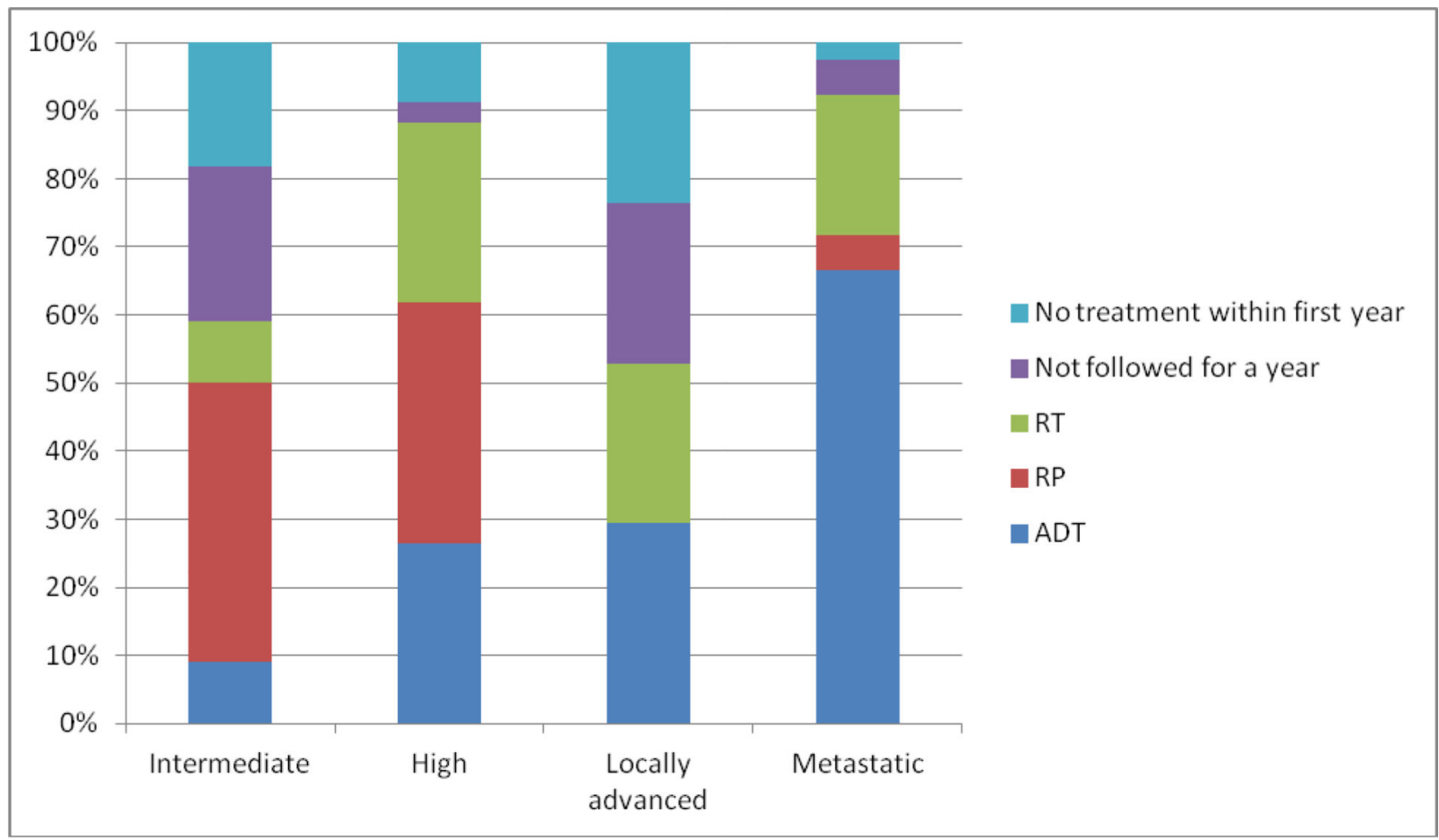

*Primary treatment defined as main treatment within first year of diagnosis: ADT = androgen deprivation therapy, $\mathrm{RP}=$ radical prostatectomy, $\mathrm{RT}$ = radiotherapy or brachytherapy. Men with neoadjuvant ADT were classified according to their main treatment.

** Risk groups defined according to the NCCN criteria as: Low risk: TO, T1c, T2a and GS $\leq 6$ and PSA < 10; Intermediate risk: T2b, T2c or GS 7 or PSA 10-20; High risk: T3a or GS 8-10 or PSA $\geq 20$; Locally advanced: T3b, T3c, T4; Metastatic: N1 or M1.

\section{Figure 2.}

Main treatment within first year, by risk group $(n=112)$ 


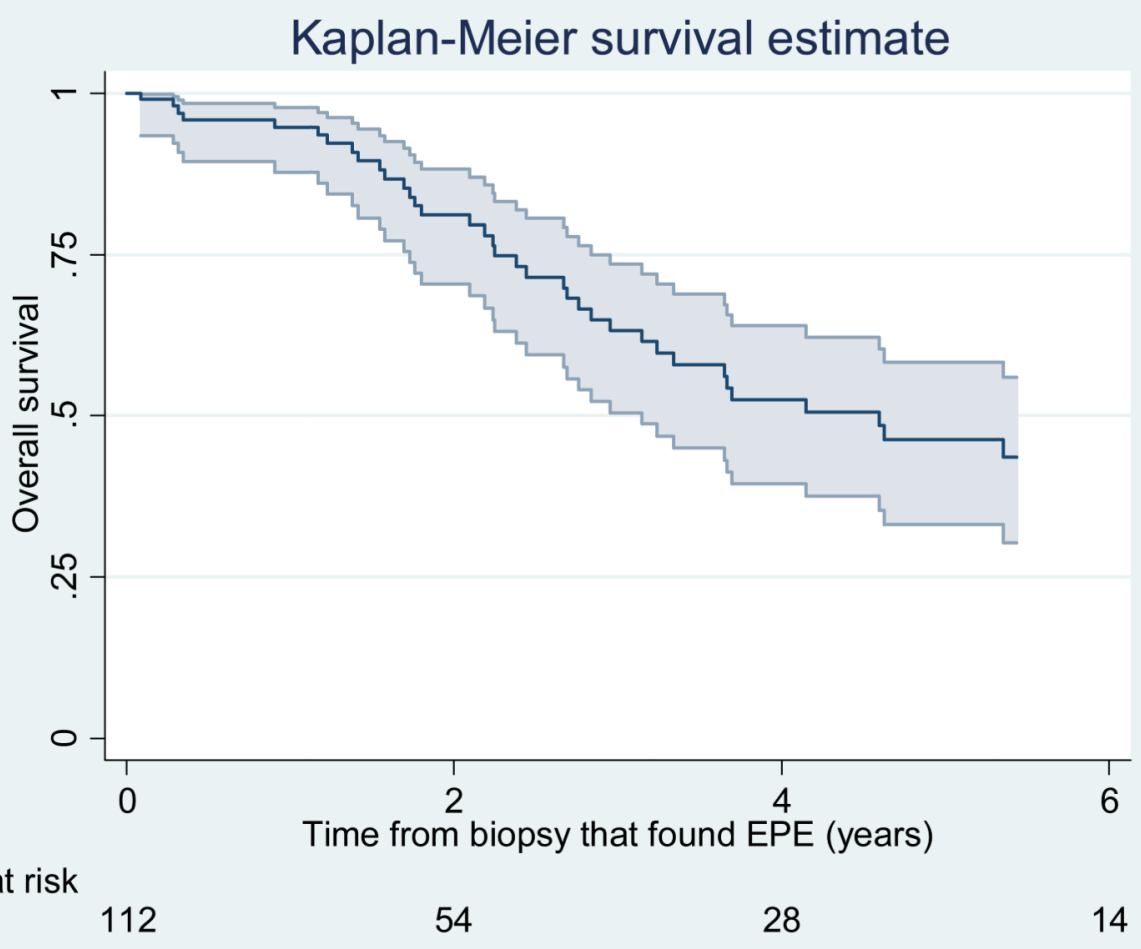

Figure 3.

Overall survival for cohort 


\section{Table 1}

\section{Patient characteristics $(\mathrm{N}=112)$}

Numbers represent $\mathrm{n}(\%)$ or median (interquartile range)

\begin{tabular}{|c|c|}
\hline Age at urology visit, years & $68(61,75)$ \\
\hline Total PSA (ng/mL) & $15.9(8.1,62.3)$ \\
\hline$<10$ & $40(36 \%)$ \\
\hline $10-20$ & $22(20 \%)$ \\
\hline $20-100$ & $30(27 \%)$ \\
\hline $100+$ & $20(18 \%)$ \\
\hline \multicolumn{2}{|l|}{ Biopsy Gleason score category } \\
\hline 6 & $1(1 \%)$ \\
\hline $3+4$ & $19(17 \%)$ \\
\hline $4+3$ & $7(6 \%)$ \\
\hline 8 & $15(13 \%)$ \\
\hline $9-10$ & $70(63 \%)$ \\
\hline \multicolumn{2}{|l|}{ Clinical $\mathrm{T}$ stage } \\
\hline $\mathrm{T} 1$ & $28(25 \%)$ \\
\hline $\mathrm{T} 2$ & $23(20.5 \%)$ \\
\hline $\mathrm{T} 3$ & $34(30 \%)$ \\
\hline $\mathrm{T} 4$ & $14(12.5 \%)$ \\
\hline TX/Missing & $13(12 \%)$ \\
\hline \multicolumn{2}{|l|}{ Risk group ${ }^{*}$} \\
\hline Low & $0(0 \%)$ \\
\hline Intermediate & $22(20 \%)$ \\
\hline High & $34(30 \%)$ \\
\hline Locally advanced & $17(15 \%)$ \\
\hline Metastatic & $39(35 \%)$ \\
\hline
\end{tabular}

Risk groups defined according to the NCCN criteria as: Low risk: T0, T1c, T2a and GS $\leq 6$ and PSA < 10; Intermediate risk: T2, T2b, T2c or GS 7 or PSA 10-20; High risk: T3, T3a or GS 8-10 or PSA 220; Locally advanced: T3b-c, T4; Metastatic: N1 or M1. 


\section{Detailed biopsy information}

Table 2

Numbers represent $\mathrm{n}(\%)$ or median (inter quartile range)

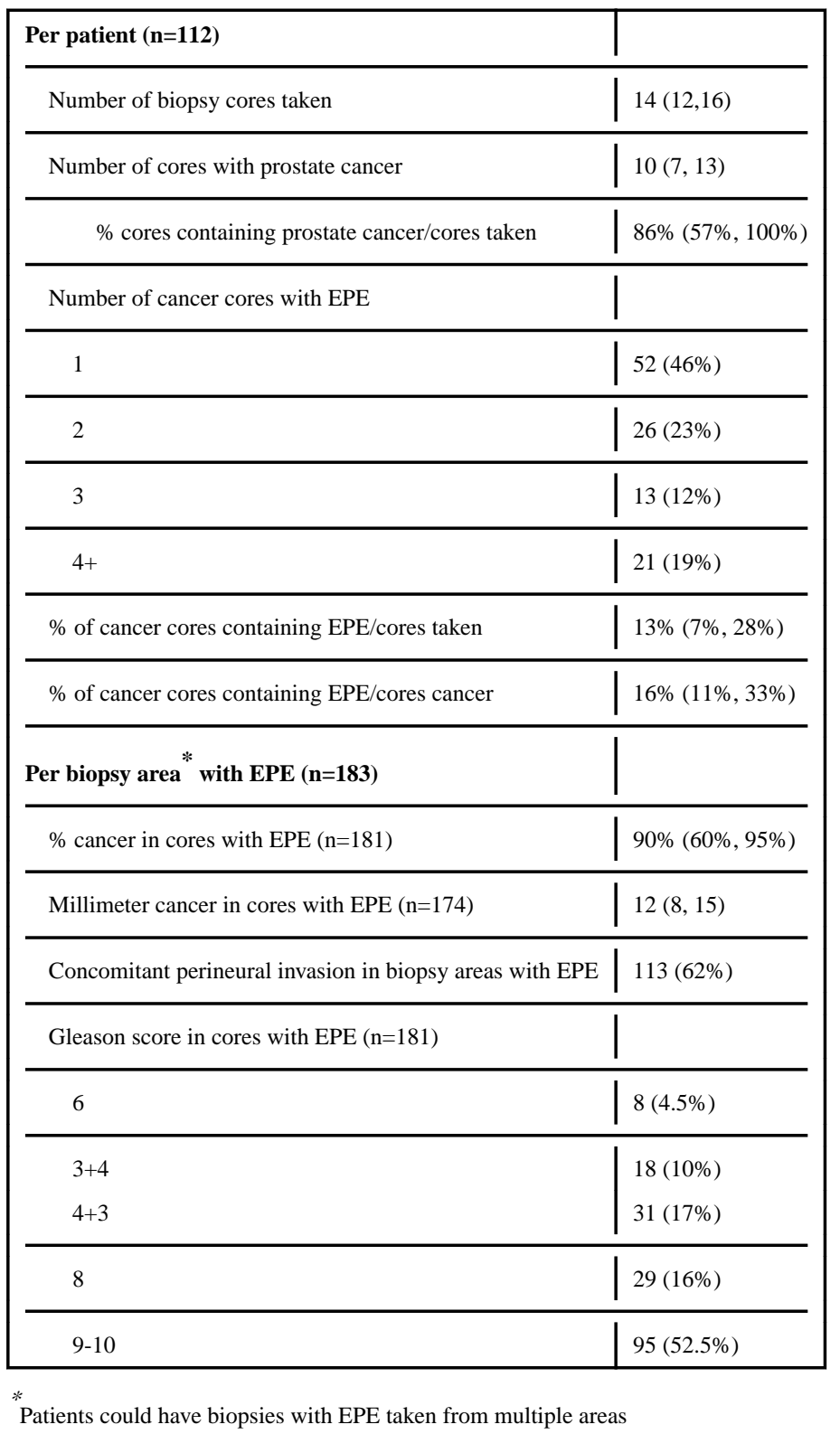

J Urol. Author manuscript; available in PMC 2017 September 01. 